\title{
siRNA delivery: current trends and future perspectives
}

\author{
"Delivery of therapeutic nucleic acids in general and small \\ interfering RNAs in particular although a relatively nascent \\ area of research, has already overcome several technical and \\ regulatory hurdles over past few years."
}

\section{Article submitted: 5 November 2015; Accepted for publication: 5 November 2015; Published online: 15 January 2016}

Delivery of therapeutic nucleic acids in general and small interfering RNAs (siRNAs) in particular although a relatively nascent area of research, has already overcome several technical and regulatory hurdles over past few years. Some lead formulations undergoing clinical evaluation against wide range of diseases from age-related macular degeneration (AMD) to hypercholesteremia, amyloidosis and cancer. Roughly 4000 diseases are associated with malfunctioning genes in a particular cell type [1]. Gene therapy provides opportunity to modify (inhibit or enhance) the expression of disease-causing genes at the cellular level. Gene correction at the DNA level poses risks of inciting mutations or teratogenicity in comparison with RNA interference (RNAi) approach, which works at the transcription level leading to desired outcomes at the protein production stage such as therapeutic gene silencing of a cancer-causing gene.

Currently, the key molecules employed in executing RNAi strategies are short hairpin RNA (shRNA), small interfering RNA (siRNA) or endogenous microRNA, of which siRNA forms the major mode presently under clinical evaluation [2].

siRNA molecules are easy to synthesize and do not need to be delivered into the nucleus as they do not require genome integration or the usage of DNA promoters [3]. They act in a sequence-specific manner against a target messenger RNA (mRNA), which avoids unintended off-target effects. The RNA-induced silencing complex (RISC), which drives
RNAi, protects siRNA from degradation by cytosolic nucleases thereby recycling the molecule for many rounds of cleavage activity against the target mRNA. This implies that even few siRNA molecules if delivered stably into the cell cytosol are sufficient to trigger long-lasting gene silencing.

In order to reach the desired organ/tissue and subsequently cross the cell membrane, these therapeutic siRNAs need to overcome several physiological barriers such as renal clearance, serum degradation and cellspecific uptake. Following crossing the cell membrane, intracellular barriers in the form of saturation of endogenous RNAi machinery or endosomal escape postendocytosis can reduce the efficiency of the delivered siRNA molecules [4]. Small negatively charged siRNAs can also lead to immunotoxicity and over the past years efforts have been dedicated to develop an efficient carrier modalities to overcome these systemic barriers and toxicity issues, which broadly falls into viral and nonviral strategies. Due to safety concerns on usage of viral vectors, nonviral techniques have taken the center-stage in siRNAs delivery.

Delivery vehicles such as liposomes, lipidbased nanoparticles (LNPs) or polymeric nanoparticles are biodegradable, have relatively low toxicity, are stable in storage, easyto scale up and have low production costs - traits favored from an industrial perspective. Moreover, most components used in the development of nanostructures in the form of lipids or polymers are approved by the

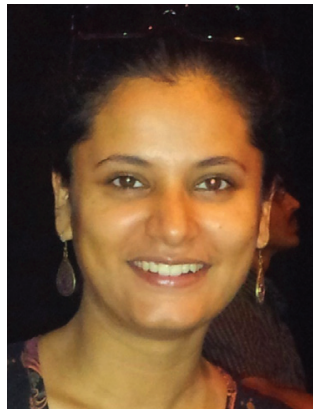

Manu Smriti Singh

Laboratory of NanoMedicine, Department of Cell Research \& Immunology, George S. Wise Faculty of Life Sciences, Tel Aviv University, Tel Aviv 69978, Israel

Department of Materials Science \& Engineering, The lby \& Aladar Fleischman Faculty of Engineering, Tel Aviv University, Tel Aviv, 69978, Israel Center for Nanoscience \& Nanotechnology, Tel Aviv University, Tel Aviv 69978, Israel

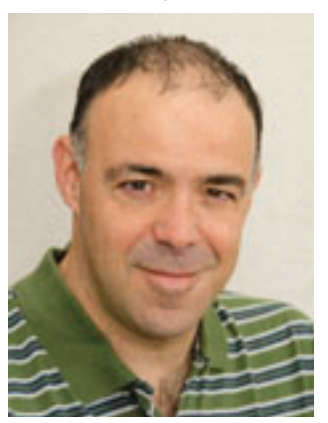

Dan Peer

Author for correspondence: Laboratory of NanoMedicine, Department of Cell Research \& Immunology, George S. Wise Faculty of Life Sciences, Tel Aviv University, Tel Aviv 69978, Israel

Department of Materials Science \&

Engineering, The lby \& Aladar Fleischman Faculty of Engineering, Tel Aviv University, Tel Aviv, 69978, Israel Center for Nanoscience \& Nanotechnology, Tel Aviv University, Tel Aviv 69978, Israel Tel.: +972 36407925 Fax: +97236405926 peer@tauex.tau.ac.il

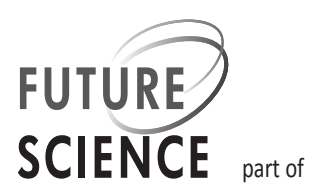


regulatory agencies for human use. From a technical standpoint, delivering siRNAs in nanocarriers offers high encapsulation rate, potentially a controllable sustained release option or burst release and stability against degradation in the biological environment [5]. In the case of cancer care, tumor physiology may aid in the accumulation of nanocarriers in the desired tissue or solid tumor via enhanced permeability and retention effect in the case of carcinoma but this needs to be tested on case-by-case studies and may not be an ultimate mechanism for all types of solid tumors.

\section{It is clear that with the ongoing progress in molecular and next-generation 'omics' technologies as well as the interdisciplinary collaborations between geneticists, material scientists, immunologists and biochemists - the future of personalized medicine will eventually materialize."}

Beginning with chemical modification of the siRNA molecules to stably sustain the harsh biological environment such as $2^{\prime} \mathrm{o}-\mathrm{Me}$ or 2'o-Flu in specific sequences, much advancement has been achieved also by using various delivery vectors. Mostly classical cationic lipids (DOTAP, DOTMA) or cationic polymers (PEI, chitosan) have been used in formulations, conjugates or complexes to form lipoplexes or polyplexes. Exosomes are also being explored for gene delivery purposes [6]. One of the most characterized platforms for siRNA delivery is the stable nucleic acid lipid particles (SNALPs), which represent approximately $70-120 \mathrm{~nm}$ nanocarriers. Many formulations under clinical investigations from Tekmira Pharmaceuticals and Alnylam Pharmaceuticals are formulated using SNALPs, which use ionizable lipids such as the DLin-DMA-based amino lipids as their prime component [7]. A common feature of most carriers is PEGylation (coating of polyethylene glycol [PEG]), which provides both steric stability and protection from systemic uptake by macrophages and other types of leukocytes. Further modifying the nanocarrier surface with molecules that can impart/enhance functional activity are for example the use of targeting moieties to endow these carriers' high selectivity toward cell surface receptors $[5,8,9]$.

\section{Progressing beyond the liver}

Much activity in the field of therapeutic gene silencing has been done with liver diseases and indications (among them liver cancers, amyloidosis and hypercholesteremia) - most likely since the SNALPs tend to target hepatocytes. It is now high time to develop strategies for targeting other cell types and demonstrate cell-specific delivery and selective gene knockdown that will be translated into therapeutic gene silencing.
Our lab has demonstrated efficient siRNA delivery and subsequent knockdown of target genes with LNPs in highly aggressive ovarian and brain cancer xenografted mouse models [10,11]. In addition, we have recently shown specific gene knockdown to a subset of circulatory $\mathrm{CD}^{+} \mathrm{T}$ cells with utmost precision [12]. This highlights the efficiency and selectivity of siRNAbased delivery platforms. With progress in knowledge at the molecular and cellular level with precision delivery strategies and coupled with next-generation sequencing, personalized therapies are coming to fore, and it is likely we will observe an increase demand of gene-specific correction therapies in the very near future with major investments from industry [13].

Important consideration for industrial involvement is the homogeneity of the nanoformulations, which can yield reproducibility with respect to particle's physicochemical and functional characterization. One way to achieve this goal is the use of microfluidics-based manufacturing platforms, which allows mixing of the formulation components in a controlled manner. One such system is the NanoAssemblr ${ }^{\mathrm{TM}}$ that offers a scalable, rapid and simple approach with minimal batchto-batch variations [14] however, analytics of each component and physicochemical characterization of the formulations are essential in order to be able to scale up these nanoformulations.

\section{Future perspective}

Although numerous formulations are being devised lately, focus should remain on biological stability, specificity and safety of these nanocarriers, which can be easily translatable from bench-to-bedside. Complex formulations are not only difficult to scale-up, they can be costly and potentially also challenging from a regulatory standpoint. Efforts should be focusing on strategies that are targeting tumors, in other words, going beyond the liver with siRNAs although the first RNAi drugs will most likely be related to diseases in the liver - utilizing the natural mechanism by which lipid-based nanoparticles (LNPs) accumulate in this organ.

Lipids form the core components of many types of nanocarriers for siRNA delivery. Combination therapies with several siRNAs targeting different survival pathways or combination of particular siRNA that may sensitize the treatment of a tumor together with anticancer drugs may become novel therapeutic modalities.

It is clear that with the ongoing progress in molecular and next-generation 'omics' technologies as well as the interdisciplinary collaborations between geneticists, material scientists, immunologists and biochemists - the future of personalized medicine will eventually materialize. Positive outcomes of much undertrial 
RNAi therapeutics will also boost the confidence of the drug industry to invest in this avenue with special emphasis on cancer care.

\section{Financial \& competing interests disclosure}

This work was supported in part by grants from the Dotan Hematology Center at Tel Aviv University, The Lewis Family Trust, Israel Science Foundation (award \#181/10), the ICORE Program of the Planning and Budgeting Committee and The Israel Science Foundation (grant number 41/11) FTA: Nano-

\section{References}

1 Gene Therapy. American Medical Association. www.ama-assn.org

2 Peer D. Harnessing RNAi nanomedicine for precision therapy. Mol. Cell. Ther. doi:10.1186/2052-8426-2-5 (eCollection 2014).

3 Wittrup A, Lieberman J. Knocking down disease: a progress report on siRNA therapeutics. Nat. Rev. Genet. 16(9), 543-552 (2015).

4 Daka A, Peer D. RNAi-based nanomedicines for targeted personalized therapy. Adv. Drug Deliv. Rev. 64(13), 1508-1521 (2012).

5 Peer D, Karp JM, Hong S, Farokhzad OC, Margalit $R$, Langer R. Nanocarriers as an emerging platform for cancer therapy. Nat. Nanotechnol. 2(12), 751-760 (2007).

6 Junquera E, Aicart E. Recent progress in gene therapy to deliver nucleic acids with multivalent cationic vectors. $A d v$. Colloid Interface Sci. doi:10.1016/j.cis.2015.07.003 (2015) (Epub ahead of print).

7 Ozcan G, Ozpolat B, Coleman RL, Sood AK, LopezBerestein G. Preclinical and clinical development of siRNAbased therapeutics. Adv. Drug Deliv. Rev. 87, 108-119 (2015). medicines for Personalized Theranostics of the Israeli National Nanotechnology Initiative and by The Leona M. and Harry B. Helmsley Nanotechnology Research Fund awarded to D Peer. $D$ Peer declares financial interests in Quiet Therapeutics. The authors have no other relevant affiliations or financial involvement with any organization or entity with a financial interest in or financial conflict with the subject matter or materials discussed in the manuscript apart from those disclosed.

No writing assistance was utilized in the production of this manuscript.

8 Lachelt U, Wagner E. Nucleic acid therapeutics using polyplexes: a journey of 50 years (and beyond). Chem. Rev. 115(19), 11043-11078 (2015).

9 Peer D, Park EJ, Morishita Y, Carman CV, Shimaoka M. Systemic leukocyte-directed siRNA delivery revealing cyclin D1 as an anti-inflammatory target. Science 319(5863), 627-630 (2008).

10 Cohen K, Emmanuel R, Kisin-Finfer E, Shabat D, Peer D. Modulation of drug resistance in ovarian adenocarcinoma using chemotherapy entrapped in hyaluronan-grafted nanoparticle clusters. ACS Nano 8(3), 2183-2195 (2014).

11 Cohen ZR, Ramishetti S, Peshes-Yaloz N et al. Localized RNAi therapeutics of chemoresistant grade IV glioma using hyaluronan-grafted lipid-based nanoparticles. ACS Nano 9(2), 1581-1591 (2015).

12 Ramishetti S, Kedmi R, Goldsmith M et al. Systemic gene silencing in primary $\mathrm{T}$ lymphocytes using targeted lipid nanoparticles. ACS Nano 9(7), 6706-6716 (2015).

13 Rosenblum D, Peer D. Omics-based nanomedicine: the future of personalized oncology. Cancer Lett. 352(1), 126-136 (2014).

14 Walsh C, Ou K, Belliveau NM et al. Microfluidic-based manufacture of siRNA-lipid nanoparticles for therapeutic applications. Methods Mol. Biol. 1141, 109-120 (2014). 